\title{
Efficacy and Safety of Intravitreal Aflibercept Injection in Japanese Patients with Neovascular Glaucoma: Outcomes from the VENERA Study
}

\author{
Masaru Inatani · Tomomi Higashide $\cdot$ Kenji Matsushita • \\ Daisuke Nagasato · Hitoshi Takagi · Mari Ueki · Yasuyuki Takai • \\ Ken Miyazaki · Yuji Iwamoto · Masato Kobayashi · Sergio Leal
}

Received: October 20, 2020 / Accepted: November 19, 2020 / Published online: December 16, 2020

(C) The Author(s) 2020

\begin{abstract}
Introduction: Neovascular glaucoma is characterized by neovascularization of the iris and anterior angle chamber. Intravitreal anti-vascular endothelial growth factor agents may decrease intraocular pressure (IOP) and improve neovascularization. The VENERA study assessed the efficacy and safety of intravitreal aflibercept (IVT-AFL) in patients with neovascular glaucoma.
\end{abstract}

Methods: This was a 5-week, single-arm, nonrandomized, open-label, phase 3 study performed at 7 study sites in Japan that enrolled

Supplementary Information The online version contains supplementary material available at https:// doi.org/10.1007/s12325-020-01580-y.

M. Inatani $(\bowtie)$

Department of Ophthalmology, Faculty of Medical Sciences, University of Fukui, Fukui, Japan

e-mail: inatani@u-fukui.ac.jp

T. Higashide

Department of Ophthalmology, Kanazawa

University Graduate School of Medical Science,

Kanazawa, Japan

K. Matsushita

Department of Ophthalmology, Osaka University

Graduate School of Medicine, Suita, Osaka, Japan

D. Nagasato

Department of Ophthalmology, Saneikai Tsukazaki

Hospital, Hyogo, Japan
Japanese patients with anterior segment neovascularization and IOP $>25 \mathrm{mmHg}$ who had not undergone (within 30 days prior), nor were imminently scheduled to undergo (within 8 days following) intraocular surgeries, including panretinal photocoagulation (PRP). Patients received background therapy plus $2 \mathrm{mg}$ IVT-AFL at baseline. Background therapy with systemic IOP-lowering drugs was prohibited for 3 days before day 1 and until IOP evaluation at week 1 . The primary endpoint was the change in IOP from baseline to week 1 and the secondary endpoint was the proportion of patients with an improvement of $\geq 1$ grade of neovascularization of the angle (NVA) from baseline to week 1 .

Results: Sixteen patients received treatment (full analysis set); the per-protocol set comprised 15 patients. The mean IOP decreased

\author{
H. Takagi \\ Department of Ophthalmology, St. Marianna \\ University School of Medicine, Kanagawa, Japan \\ M. Ueki \\ Department of Ophthalmology, Osaka Medical \\ College, Takatsuki, Osaka, Japan \\ Y. Takai \\ Department of Ophthalmology, Faculty of \\ Medicine, Shimane University, Shimane, Japan \\ K. Miyazaki · Y. Iwamoto · M. Kobayashi \\ Bayer Yakuhin, Ltd., Osaka, Japan \\ S. Leal \\ Bayer Consumer Care AG, Basel, Switzerland
}


from $34.1 \mathrm{mmHg}$ at baseline to $25.8 \mathrm{mmHg}$ at week 1 (mean change, $-8.3 \mathrm{mmHg}$ [95\% confidence interval; $\mathrm{CI}-12.2$ to $-4.4 ; P=0.0004]$ ). At week 1, 81.3\% of patients had an improvement in the grade of neovascularization of the iris (NVI) and $50.0 \%$ of patients had an improvement in NVA grade. The proportion of patients with controlled IOP $(\leq 21 \mathrm{mmHg})$ was $43.8 \% \quad(95 \%$ CI $19.8-70.1)$ at week 1 , and increased to $56.3 \%$ at week 2 and $86.7 \%$ at week 5. The most common ocular treatment-emergent adverse event was eye pain, which occurred in 4 patients $(25.0 \%)$.

Conclusions: IVT-AFL was associated with statistically significant and clinically meaningful IOP reductions, without concomitant use of systemic IOP-lowering drugs or PRP. The safety profile was consistent with the known safety profile of IVT-AFL. These findings supplement those from the previous VEGA study, and suggest that IVT-AFL may be a potential treatment option for patients with neovascular glaucoma. Trial Registration: Clinicaltrials.gov identifier NCT03639675.

Keywords: Anti-vascular endothelial growth factor; Anti-VEGF; Intraocular pressure; Intravitreal aflibercept; Neovascular glaucoma; Neovascularization of the angle; Neovascularization of the iris; NVG

\section{Key Summary Points}

Why carry out this study?

Retinal ischemia, thought to be the mediator in most cases of neovascular glaucoma, results in the release of angiogenic factors, such as vascular endothelial growth factor (VEGF), which trigger neovascularization.

It has been suggested that intravitreal antiVEGF agents may improve intraocular pressure (IOP) and neovascularization in patients with neovascular glaucoma.
The VENERA study was designed to assess the efficacy and safety of intravitreal aflibercept (IVT-AFL) in patients with neovascular glaucoma in the absence of systemic IOP-lowering drugs and panretinal photocoagulation.

What was learned from the study?

Patients who received IVT-AFL (without systemic IOP-lowering drugs) showed a statistically and clinically significant decrease in mean IOP (mean change, $-8.3 \mathrm{mmHg}$ at week 1 ), and an improvement in neovascularization of the iris and angle $(81.3 \%$ and $50.0 \%$ improved at week 1 , respectively).

The incidence of treatment-emergent adverse events was consistent with the known safety profile for IVT-AFL.

These findings support the role of antiVEGF agents, specifically IVT-AFL, in managing patients with neovascular glaucoma.

\section{DIGITAL FEATURES}

This article is published with digital features, including a summary slide, to facilitate understanding of the article. To view digital features for this article go to https://doi.org/10.6084/ m9.figshare.13252121.

\section{INTRODUCTION}

Neovascular glaucoma (NVG) is a secondary ocular condition that most commonly occurs in patients with proliferative diabetic retinopathy (PDR), ischemic central retinal vein occlusion, and ocular ischemic syndrome [1, 2]. The condition, characterized by the development of neovascularization of the iris (NVI) and neovascularization of the angle (NVA), prevents normal drainage from the anterior segment of the eye and leads to elevated intraocular 
pressure (IOP) $[3,4]$. The main therapy for NVG is panretinal photocoagulation (PRP), which causes shrinkage of the abnormal blood vessels [4]. However, because the regression of neovascularization is not always immediate, patients may need close monitoring and combined local and systemic treatment to control IOP for several weeks [5].

Elevated vascular endothelial growth factor (VEGF) plays a major role in mediating active intraocular neovascularization in patients with ischemic retinal diseases, and high levels of VEGF are present in patients with NVG $[2,6]$. Intravitreal aflibercept (IVT-AFL) injection $(2 \mathrm{mg}$ ) was approved in 2020 as a treatment for patients with NVG in Japan [7]. Results from case series suggest that anti-VEGF treatments may have a positive influence on outcomes in patients with NVG [8-10]. In addition, clinical guidelines for glaucoma issued by the Japanese Glaucoma Society state that intraoperative or postoperative complications can be suppressed by intravitreal injection of anti-VEGF therapy preoperatively when conducting surgeries for NVG [11].

The VEGA study was the first randomized, double-masked, sham-controlled study to assess the short-term efficacy and safety of IVT-AFL injections in Japanese patients with NVG treated with IOP-lowering therapy. Findings showed that, despite not meeting the primary endpoint, IVT-AFL was associated with clinically meaningful improvements in IOP control and may offer a potential treatment option for this patient group [12]. The authors hypothesized that the use of systemic IOP-lowering agents during the VEGA study might have impacted the outcomes, and the current study has been designed to test this hypothesis. Here we report the results of VENERA, a single-arm, open-label study to assess the efficacy and safety of IVT-AFL in Japanese patients with NVG, excluding the influence of systemic IOP-lowering drugs and PRP.

\section{METHODS}

\section{Study Design}

VENERA (NCT03639675) was a 5-week, multicenter, single-arm, nonrandomized, open-label, phase 3 study to evaluate the efficacy and safety of IVT-AFL in patients with NVG. The study was conducted at 7 sites in Japan between October 3, 2018 and March 14, 2019. The study was conducted in accordance with the Declaration of Helsinki and the International Council for Harmonization guideline E6: Good Clinical Practice. Institutional review board approval of the protocol was obtained at each site (see supplementary material), and all patients provided written informed consent.

Eligible patients with an IOP $>25 \mathrm{mmHg}$ at screening participated in a run-in phase that involved treatment with a combination of $\geq 3$ topical IOP-lowering drugs. The topical drugs used during the run-in phase were continued unchanged until the IOP measurement was performed at week 1, after which treatment could be reduced according to the investigator's judgment. Treatment with systemic IOP-lowering drugs was prohibited for 3 days before day 1 and until IOP evaluation at week 1 . Systemic use of anti-VEGF drugs and topical ophthalmic atropine sulfate hydrate were prohibited during the period of the study.

During the run-in period, IOP was re-evaluated $1 \mathrm{~h}( \pm 10 \mathrm{~min})$ after the administration of the topical IOP-lowering drugs. If no decrease was evident compared with screening, that value was considered the baseline IOP measurement. If a decrease in IOP compared with screening was detected, the patient was reevaluated $2 \mathrm{~h}( \pm 10 \mathrm{~min})$ after the start of the run-in phase. If the IOP was $>25 \mathrm{mmHg}$ after $2 \mathrm{~h}$, that measurement was considered the baseline measurement. If IOP decreased to $25 \mathrm{mmHg}$ or lower between screening and planned treatment, the patient was considered a screening failure. At baseline, patients received IVT-AFL $2 \mathrm{mg}$ (eFig. 1 in the supplementary material).

Patients were evaluated by gonioscopy to determine development of NVI and NVA in conjunction with slit-lamp microscopy. NVI and NVA were assessed in the study eye using the NVI and NVA grading systems [13] on a scale of 0 to 4 (eTable 1 in the supplementary material). IOP was assessed using applanation tonometry (Goldmann [standard technique] or 
Tono-Pen [Reichert]). Noncontact tonometry was not permitted.

\section{Patients}

Japanese patients aged $\geq 20$ years were eligible for inclusion if they had a diagnosis of NVG with neovascularization in the anterior segment (both iris and anterior chamber angle) and IOP $>25 \mathrm{mmHg}$ due to neovascularization in the study eye. Key exclusion criteria were angle closure due to conditions other than NVG or a known or suspected ocular or periocular infection. Patients who had received intraocular administration of anti-VEGF drugs in the study eye within 60 days before day 1 were excluded, as were those who received systemic IOP-lowering drugs in the 3 days before day 1. Patients who had undergone other intraocular surgeries, including PRP, in the study eye within 30 days before day 1 or who were scheduled to undergo surgery, including PRP, during the 8 days after day 1 were also excluded. Full exclusion criteria are provided in the online supplementary material.

\section{Endpoints}

The primary endpoint was the mean change in IOP from baseline to week 1 (pre-injection IOP evaluation). After baseline, IOP was measured within $\pm 2 \mathrm{~h}$ of the measurement time at baseline to minimize any impact of diurnal IOP variations. The secondary endpoint was the proportion of patients who had an improved NVI grade (by $\geq 1$ grade) from baseline to week 1 . Exploratory endpoints were the change in IOP from baseline by visit, the proportion of patients with NVA change of $\geq 1$ grade from baseline to week 1 , and the proportion of patients who achieved IOP $\leq 21 \mathrm{mmHg}$ at week 1. Safety was evaluated throughout the study.

\section{Statistical Analysis}

Based on a mean (standard deviation [SD]) change in IOP from baseline to predose at week 1 of $-8.0(9.0) \mathrm{mmHg}$, a sample size of 16 patients was calculated to have $>90 \%$ power to detect an upper limit of 2 -sided $95 \%$ confidence interval (CI) (by 1-sample $t$ statistics) of mean change in IOP from baseline to predose level at week 1 was less than the predetermined threshold of $0 \mathrm{mmHg}$ (assumed change in IOP if untreated). The per-protocol set (PPS) was the primary analysis set for efficacy analyses and included all patients who received IVT-AFL on day 1 , had a baseline IOP measurement and $\geq 1$ postbaseline IOP measurement before or at week 1 , and showed no validity findings that might confound efficacy assessments. The efficacy variables measured within 4 days of the IVT-AFL dose (day 4 included) were excluded from the PPS analysis because, in line with its mechanism of action, IVT-AFL might require a few days to take effect. The postbaseline IOP, which was measured within $\pm 2 \mathrm{~h}$ of the time the measurement was taken at baseline, was excluded from analyses in the PPS to avoid diurnal variation of IOP. The full analysis set (FAS) included all patients who received IVTAFL on day 1, had a baseline IOP measurement, and had $\geq 1$ postbaseline IOP measurement before or at week 1 . The FAS was used for supplemental analyses of efficacy. The safety analysis set (SAS) included all patients who were assigned to receive and did receive $\geq 1$ injection of IVT-AFL.

For the primary efficacy analysis, the change in IOP from baseline at week 1 was summarized descriptively in the PPS. The point estimate and 2-sided 95\% CI using 1-sample $t$ statistics were calculated. For the secondary efficacy analysis, the proportion of patients who had an improved NVI grade from baseline at week 1 was summarized descriptively. The point estimate and its 2-sided 95\% CI using the Clopper-Pearson method were calculated. Improvement by $\geq 1$ grade from baseline was categorized as "improved". Analysis of categorical variables was performed using frequency tables, and continuous variables were analyzed using summary statistics (mean, standard deviation, minimum, median, and maximum). Statistical evaluation was performed by using the software package SAS version 9.2 or higher (SAS Institute Inc., Cary, NC). 


\section{RESULTS}

\section{Patients}

A total of 17 patients were screened; 16 patients had an IOP measurement at baseline and at week 1 and received 1 IVT-AFL injection. Fifteen patients completed the follow-up period; 1 patient withdrew (withdrawal by patient) from the study. All patients were Japanese and most (75.0\%) were male. Patients had a mean (SD) age of 65.6 (12.9) years (Table 1). The combination of topical IOP-lowering drugs used at baseline (Table 1) did not change during the study. In total, 3 patients received IOP-lowering treatment (acetazolamide), 1 patient received PRP, and 1 patient received PRP and underwent surgery (trabeculectomy) after week 1.

\section{Efficacy}

The mean (SD) IOP decreased from 34.1 (6.7) $\mathrm{mmHg}$ at baseline to $25.8(8.0) \mathrm{mmHg}$ at week 1 . The primary endpoint, the mean change in IOP from baseline to week 1 , was $-8.3 \mathrm{mmHg} \quad(95 \% \quad \mathrm{CI}-12.2$ to -4.4 ; $P=0.0004)$. The decrease in mean IOP per visit up to week 5 is presented in Fig. 1. The proportion of patients with controlled IOP $(\leq 21 \mathrm{mmHg})$ was $43.8 \%$ (95\% CI 19.8-70.1) at week 1 , and increased to $56.3 \%$ at week 2 and to $86.7 \%$ at week 5 (Fig. 2).

\section{NVI and NVA}

The proportion of patients whose NVI grade improved, remained stable, or worsened from baseline to week 1 is presented in Fig. $3 \mathrm{a}$. The secondary endpoint, change in NVI grade from baseline, was improved in $81.3 \%$ of patients at week 1 and $86.7 \%$ of patients at week 5 . The proportion of patients with improvement in NVA grade from baseline was $50.0 \%$ at week 1 (Fig. 3 b) and $66.7 \%$ at week 5 .
Table 1 Patient baseline characteristics and demographics (PPS)

\begin{tabular}{ll}
\hline & $\begin{array}{l}\text { IVT-AFL } \\
\boldsymbol{N}=\mathbf{1 6}\end{array}$ \\
\hline Mean age, years & $65.6(12.9)$ \\
Male, $n$ (\%) & $12(75.0)$ \\
Primary diagnosis, $n$ (\%) & \\
Proliferative diabetic retinopathy & $10(62.5)$ \\
Central retinal vein occlusion & $3(18.8)$ \\
Ocular ischemic syndrome & $1(6.3)$ \\
Other & $2(12.5)$ \\
Duration of disease, days & $60.8(133.7)$ \\
Stage of NVG, $n$ (\%) & \\
Open angle & $16(100.0)$ \\
Closed angle & 0 \\
IOP, mmHg & $34.1(6.7)$ \\
NVI grade 3 or 4 (pooled), $n(\%)$ & $5(31.3)$ \\
NVA grade 3 or 4 (pooled), $n(\%)$ & $9(56.3)$ \\
Topical IOP-lowering treatment, $n(\%)$ & \\
Prostaglandin analogue & $16(100.0)$ \\
Sympatholytic agent & $15(93.8)$ \\
Carbonic anhydrase inhibitor & $15(93.8)$ \\
Sympathomimetic agent & $7(43.8)$ \\
Rho kinase inhibitor & $6(37.5)$ \\
\hline V &
\end{tabular}

Values are mean $(\mathrm{SD})$ unless otherwise stated $I O P$ intraocular pressure, $I V T-A F L$ intravitreal aflibercept, $N V A$ neovascularization of the angle, $N V G$ neovascular glaucoma, NVI neovascularization of the iris, PPS perprotocol set, $S D$ standard deviation

\section{Safety}

Treatment-emergent adverse events (TEAEs) during the study were reported in 6 patients (37.5\%) (Table 2). The most common ocular TEAE was eye pain $(25.0 \%)$, and corneal erosion and eye pruritus were observed in 1 patient each. At least 1 TEAE was observed in 6 patients 


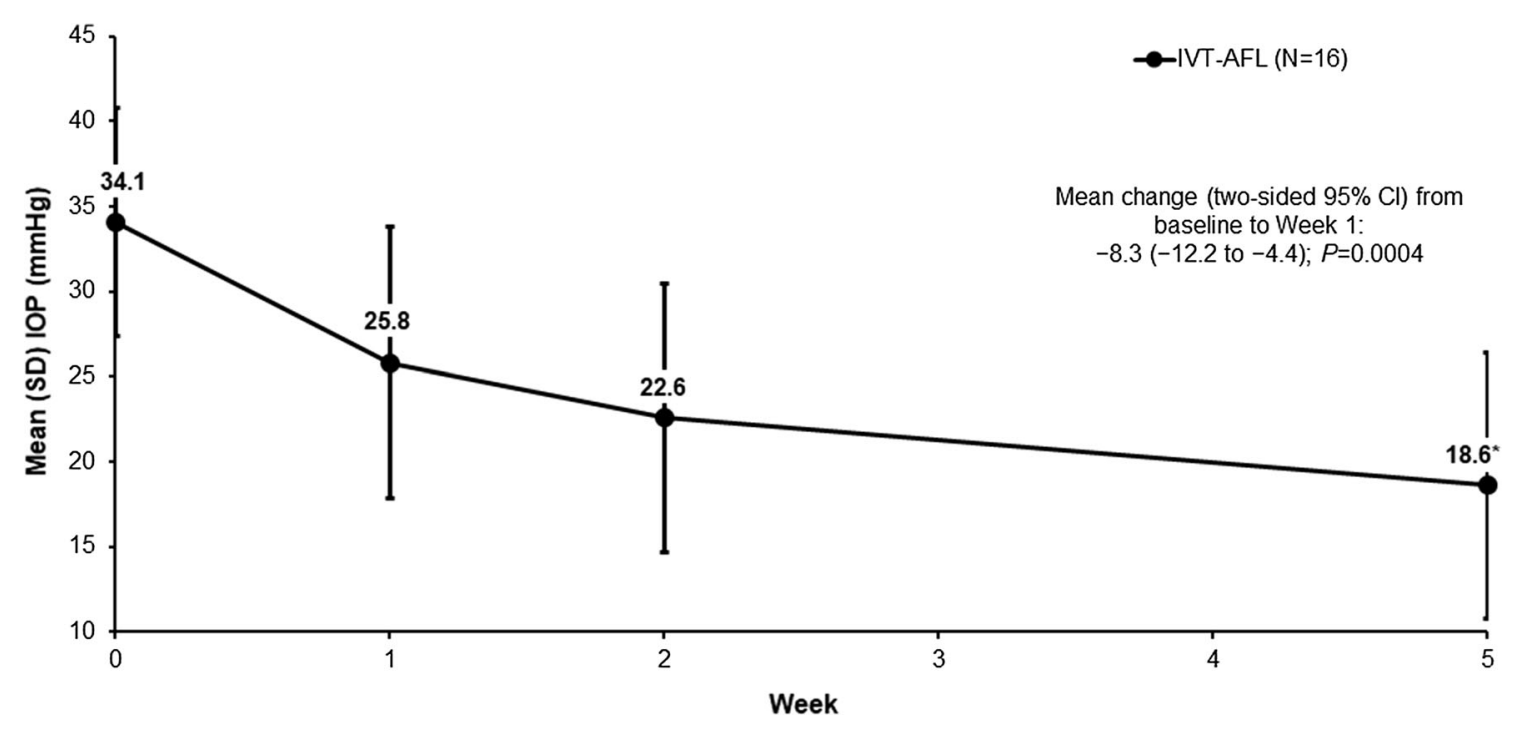

Fig. 1 Mean IOP $(\mathrm{mmHg})$ from baseline to week 5 (PPS). ${ }^{*} n=15$. The 2 -sided $95 \% \mathrm{CI}$ and $P$ value were estimated using 1-sample $t$ statistics. 3 patients received acetazolamide and 2 patients underwent surgery after evaluation

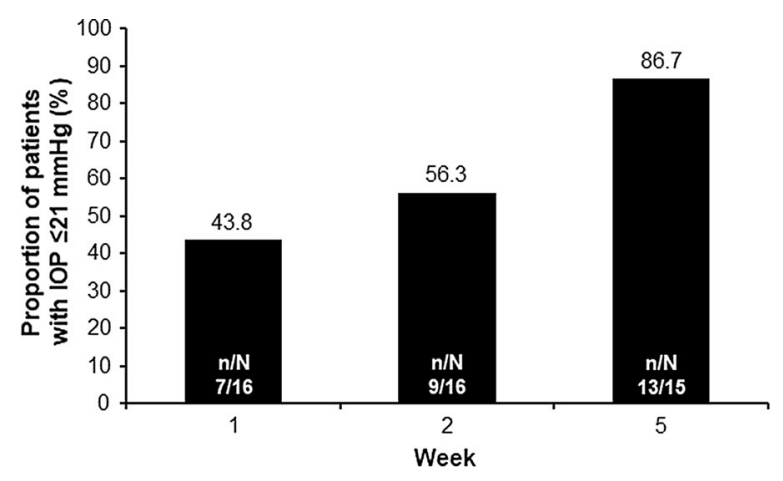

Fig. 2 Proportion of patients with IOP $\leq 21 \mathrm{mmHg}$ per visit up to week 5 (PPS). IVT-AFL intravitreal aflibercept, PPS per-protocol set

(37.5\%), 1 of which was considered related to treatment (headache), and 2 were related to injection procedure (eye pain and eye pruritus). TEAEs were moderate $(n=2,12.5 \%)$ or mild $(n=4,25.0 \%)$. A serious TEAE was reported in 1 patient (6.3\%). This patient experienced pneumonia considered unrelated to IVT-AFL treatment or the injection procedure. No Antiplatelet Trialists' Collaboration (APTC)-defined arterial thromboembolic events and no deaths were reported during the study. at week 1. IOP intraocular pressure, IVT-AFL intravitreal aflibercept, $P P S$ per-protocol set, $S D$ standard deviation

\section{DISCUSSION}

The results of the VENERA study showed that treatment with IVT-AFL resulted in a clinically meaningful and sustained reduction in IOP in Japanese patients with NVG without the influence of systemic IOP-lowering drugs or PRP. A statistically significant IOP decrease at week 1 $(-8.3 \mathrm{mmHg} \quad[95 \% \quad$ CI $\quad-12.2$ to -4.4 , $P=0.0004])$ was reported after treatment with IVT-AFL, and the improvements in efficacy shown at week 1 were observed through week 5 .

VEGA was the first phase 3 randomized study conducted to investigate the treatment of NVG with IVT-AFL or control (sham) injection. Findings showed that the mean change in IOP from baseline to week 1 was clinically meaningful $(-9.9 \mathrm{mmHg}$ in the IVT-AFL group and $-5.0 \mathrm{mmHg}$ in the sham/IVT-AFL group), although the mean difference narrowly missed statistical significance $\quad(-4.9 \mathrm{mmHg} ; \quad 95 \%$ CI -10.2 to $0.3 ; P=0.06)$. The higher than expected reduction in IOP in the sham group was likely due to concomitant use of systemic IOP-lowering drugs to prevent vision loss. Although the difference did not achieve statistical significance in the primary analysis, the 


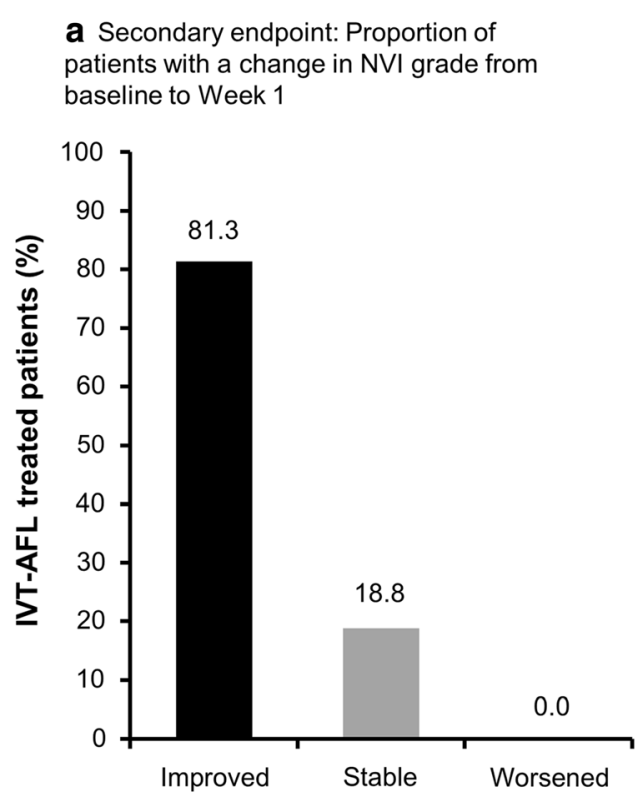

Fig. 3 Proportion of patients with improved, maintained, or worsened NVI (a) and NVA (b) grade from baseline to week 1 (PPS). Improved $=$ improvement by $\geq 1$ grade from baseline; stable $=$ no change in grade from baseline;

prespecified sensitivity analysis showed a statistically significant difference. In VEGA, the use of systemic IOP-lowering treatments was permitted, and it was concluded that this could have negatively influenced the efficacy assessment [12]. As a result, it was necessary to confirm these findings in another study. However, prohibiting the use of systemic IOP-lowering drugs in a sham treatment arm could put the safety of study participants at risk and would be considered ethically unacceptable; therefore, the VENERA study was designed to confirm the effect of IVT-AFL in the absence of the influence of systemic IOP-lowering drugs and PRP on IOP.

NVI and NVA are accompanied by development of fibrovascular membrane on the anterior surface of the iris and the iridocorneal angle of the anterior chamber, resulting in the formation of anterior synechiae and angle closure $[1,3]$. This ultimately leads to mechanical blockage of the aqueous humor outflow through the trabecular meshwork, and an increase in IOP $[1,3]$. In the VENERA study, $81 \%$ of patients showed a clinically significant improvement in NVI grade from baseline to

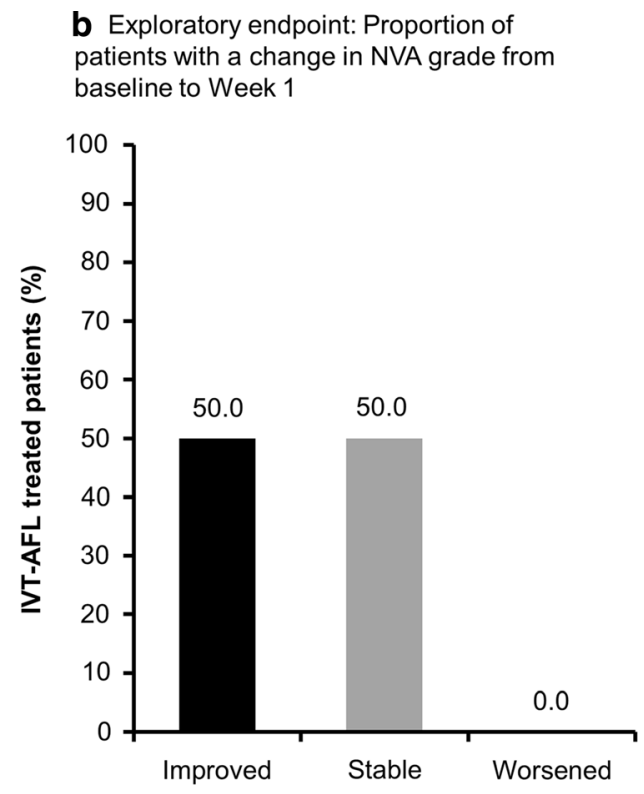

worsened $=$ worsened by $\geq 1$ grade from baseline. $I V T$ - $A F L$ intravitreal aflibercept, $N V A$ neovascularization of the angle, $P P S$ per-protocol set

week 1 after treatment with IVT-AFL. This improvement continued until week 5 (87\% of patients). An improvement in NVA grade was also observed in the current study. Results of the phase 3, randomized VEGA study showed that a larger proportion of patients in the IVT-AFL group had regression of NVI and NVA compared with patients given sham treatment [12]. Other than this, the majority of studies with antiVEGF therapy for the treatment of patients with NVG have included smaller numbers. Case series studies have shown the benefits of treatment with ranibizumab $[8,9]$ in lowering IOP or regression of neovascularization. One case series of 10 patients with NVG found that adjuvant treatment with ranibizumab led to a rapid and significant reduction in mean IOP from baseline to day 14, which was sustained at 12-month follow-up [9]. A further case series study, involving 18 patients with an IOP $\geq 25 \mathrm{mmHg}$ despite treatment with the maximum medical therapy, showed controlled IOP with 2.7 injections of ranibizumab without additional surgery in 10 patients [8]. A prospective case series of IVT-AFL in newly diagnosed NVG showed rapid 
Table 2 Safety overview at week 5 (SAS)

\begin{tabular}{|c|c|}
\hline & $\begin{array}{l}\text { IVT-AFL } \\
N=16\end{array}$ \\
\hline Any TEAE & $6(37.5)$ \\
\hline Any IVT-AFL- or IVT procedure-related AE & $3(18.8)$ \\
\hline Any IVT-AFL-related $\mathrm{AE}^{\mathrm{a}}$ & $1(6.3)$ \\
\hline Any IVT procedure-related $\mathrm{AE}^{\mathrm{b}}$ & $2(12.5)$ \\
\hline Any protocol-required procedure-related $\mathrm{AE}$ & 0 \\
\hline \multicolumn{2}{|l|}{ Ocular TEAEs $\geq 2$ patients } \\
\hline Eye pain & $4(25.0)$ \\
\hline \multicolumn{2}{|l|}{ Maximum intensity of any $\mathrm{AE}$} \\
\hline Mild & $4(25.0)$ \\
\hline Moderate & $2(12.5)$ \\
\hline Severe & 0 \\
\hline Any $S_{A E}^{c}$ & $1(6.3)$ \\
\hline $\begin{array}{l}\text { Any IVT-AFL-related or IVT procedure- } \\
\text { related SAE }\end{array}$ & 0 \\
\hline Any IVT-AFL-related AE & 0 \\
\hline $\begin{array}{l}\text { Discontinuation of study drug because of an } \\
\text { AE }\end{array}$ & 0 \\
\hline Any death & 0 \\
\hline
\end{tabular}

Values are number of patients (\%)

$A E$ adverse event, $I V T-A F L$ intravitreal aflibercept, $I V T$ intravitreal, $S A E$ serious adverse event, $S A S$ safety analysis set, TEAE treatment-emergent adverse event

${ }^{a}$ IVT-AFL-related AE was headache

b IVT procedure-related AEs were eye pain $(n=2$; $12.5 \%)$ and eye pruritus $(n=1 ; 6.3 \%)$

c SAE was pneumonia and was not considered related to treatment or injection procedure

regression of NVI and NVA, with stabilization or reduction of IOP at 52 weeks [10]. The VENERA study provides evidence of a beneficial effect of IVT-AFL for lowering IOP and regression of NVA and NVI.

Overall, the results of the VENERA study largely replicated the findings of the VEGA study and showed a significant impact of IVT-AFL in patients without the influence of systemic IOP-lowering drugs and PRP. Both studies showed a rapid decrease of IOP after a single IVT-AFL treatment, which was sustained until the end of the study with minimum need for retreatment, a finding that achieved statistical significance in the VENERA study. The safety profile was consistent with the known safety profile of IVT-AFL.

The limitations of the VENERA study include that it was a nonrandomized study with no comparator arm and involved small patient numbers. However, the study was conducted in response to findings from a previous study and required few patients to investigate whether the use of systemic IOP-lowering agents during the VEGA study might have impacted the outcomes [12]. In addition, we should acknowledge a potential impact of regression to the mean, since patients were required to have IOP $>25 \mathrm{mmHg}$ to warrant treatment with an anti-VEGF agent in this study.

\section{CONCLUSION}

The VENERA study supplements findings from the earlier VEGA study [12], and reports that treatment with IVT-AFL achieved clinically meaningful ( $>20 \%$ ) IOP reductions in the absence of systemic IOP-lowering drugs or concomitant PRP in Japanese patients with NVG and may offer a suitable treatment option for the management of some patients with NVG. In addition, IVT-AFL was associated with regression of anterior segment neovascularization (NVI/NVA improvements). The safety profile of IVT-AFL was consistent with its known safety profile.

\section{ACKNOWLEDGEMENTS}

The authors thank all the patients and investigators who contributed to this study.

Funding. Funding for the study, medical writing and editorial assistance for this manuscript, and funding for the Rapid Service Fee and Open Access Fee was provided by Bayer 
Consumer Care AG, Basel, Switzerland. In conjunction with the VENERA steering committee, Bayer participated in the design of the study; analysis and interpretation of the data; preparation, review, and approval of the manuscript; and decision to submit the manuscript for publication. Additionally, Bayer was responsible for the conduct of the study and oversight of the collection and management of data.

Medical Writing and Editorial Assistance. Medical writing and editorial support for the preparation of this manuscript (under the guidance of the authors) was provided by Louise Brady, PhD, of ApotheCom (UK), funded by Bayer Consumer Care AG, Basel, Switzerland.

Authorship. All named authors meet the International Committee of Medical Journal Editors (ICMJE) criteria for authorship for this article, take responsibility for the integrity of the work as a whole, and have given their approval for this version to be published.

Authorship Contributions. All authors contributed to the design; data acquisition, analysis, and interpretation; and preparation and final review of the manuscript. All authors approved the manuscript for submission.

Prior Presentation. The results of the VENERA study have been presented in part at the virtual 124th Annual Meeting of the Japanese Ophthalmological Society meeting (online) April 27-May 10, 2020 and EURETINA 2020 Virtual, 2-4 October, 2020.

Disclosures. Dr Masaru Inatani reports nonfinancial support from Bayer during the conduct of the study and personal fees from Bayer outside the submitted work. Dr Tomomi Higashide reports receiving personal fees from Bayer. Dr Kenji Matsushita reports receiving travel fees from Bayer Yakuhin and Senju and lecture fees from Alcon, Alcon Pharmaceutical, Kowa, Nitten, Otsuka, Pfizer, Santen, Senju, Maruho, and Bayer Yakuhin. Dr Daisuke Nagasato reports no conflicts of interest. Dr Hitoshi Takagi reports research funding from Novartis, Senju, Bayer, and Chugai and scholarship grants from Santen,
Senju, Hoya, Pfizer, Alcon Japan, Kowa, AMO Japan, and Otsuka. Dr Yasuyuki Takai and Dr Mari Ueki report no conflicts of interest. Mr Ken Miyazaki, Mr Yuji Iwamoto, and Dr Masato Kobayashi are employees of Bayer Yakuhin, Ltd., Osaka, Japan. Dr Sergio Leal is an employee of Bayer Consumer Care AG, Basel, Switzerland, and reports a patent pending (WO2018/229034).

Compliance with Ethics Guidelines. The study was conducted in accordance with the Declaration of Helsinki and the International Council for Harmonization guideline E6: Good Clinical Practice. Institutional review board approval of the protocol was obtained at each site (see supplementary material), and all patients provided written informed consent.

Data Availability. Availability of the data underlying this publication will be determined according to Bayer's commitment to the EFPIA/ PhRMA, "Principles for responsible clinical trial data sharing." This pertains to scope, time point, and process of data access. As such, Bayer commits to sharing, upon request from qualified scientific and medical researchers, patientlevel clinical trial data, study-level clinical trial data, and protocols from clinical trials in patients for medicines and indications approved in the United States (US) and European Union (EU) as necessary for conducting legitimate research. This applies to data on new medicines and indications that have been approved by the EU and US regulatory agencies on or after January 1, 2014. Interested researchers can use www. clinicalstudydatarequest.com to request access to anonymized patient-level data and supporting documents from clinical studies to conduct further research that can help advance medical science or improve patient care. Information on the Bayer criteria for listing studies and other relevant information is provided in the Study sponsors section of the portal. Data access will be granted to anonymized patient-level data, protocols and clinical study reports after approval by an independent scientific review panel. Bayer is not involved in the decisions made by the independent review panel. Bayer 
will take all necessary measures to ensure that patient privacy is safeguarded.

Open Access. This article is licensed under a Creative Commons Attribution-NonCommercial 4.0 International License, which permits any non-commercial use, sharing, adaptation, distribution and reproduction in any medium or format, as long as you give appropriate credit to the original author(s) and the source, provide a link to the Creative Commons licence, and indicate if changes were made. The images or other third party material in this article are included in the article's Creative Commons licence, unless indicated otherwise in a credit line to the material. If material is not included in the article's Creative Commons licence and your intended use is not permitted by statutory regulation or exceeds the permitted use, you will need to obtain permission directly from the copyright holder. To view a copy of this licence, visit http://creativecommons.org/licenses/by$\mathrm{nc} / 4.0 /$.

\section{REFERENCES}

1. Hayreh SS. Neovascular glaucoma. Prog Retin Eye Res. 2007;26(5):470-85.

2. Rodrigues GB, Abe RY, Zangalli C, et al. Neovascular glaucoma: a review. Int J Ret Vitreous. 2016;2:26.

3. Olmos LC, Lee RK. Medical and surgical treatment of neovascular glaucoma. Int Ophthalmol Clin. 2011;51(3):27-36.

4. Simha A, Braganza A, Abraham L, Samuel P, Lindsley K. Anti-vascular endothelial growth factor for neovascular glaucoma. Cochrane Database Syst Rev. 2013;10:CD007920.
5. Andres-Guerrero V, Perucho-Gonzalez L, GarciaFeijoo J, et al. Current perspectives on the use of anti-VEGF drugs as adjuvant therapy in glaucoma. Adv Ther. 2017;34(2):378-95.

6. Aiello LP, Avery RL, Arrigg PG, et al. Vascular endothelial growth factor in ocular fluid of patients with diabetic retinopathy and other retinal disorders. N Engl J Med. 1994;331(22):1480-7.

7. Pharmaceuticals and Medical Devices Agency. March 25 2020. https://www.info.pmda.go.jp/go/ pack/1319405A1027_1_12/?view=frame\&style= XML\&lang=ja.

8. Shen X, Chen Y, Wang Y, Yang L, Zhong Y. Intravitreal ranibizumab injection as an adjuvant in the treatment of neovascular glaucoma accompanied by vitreous hemorrhage after diabetic vitrectomy. J Ophthalmol. 2016;2016:4108490.

9. Luke J, Nassar K, Luke M, Grisanti S. Ranibizumab as adjuvant in the treatment of rubeosis iridis and neovascular glaucoma-results from a prospective interventional case series. Graefes Arch Clin Exp Ophthalmol. 2013;251(10):2403-13.

10. SooHoo JR, Seibold LK, Pantcheva MB, Kahook MY. Aflibercept for the treatment of neovascular glaucoma. Clin Exp Ophthalmol. 2015;43(9):803-7.

11. Tanihara $H$, Aihara $M$, Inatani $M$, Inoue $T$, Kiuchi YKS. The Japan Glaucoma Society guidelines for glaucoma. Nippon Ganka Gakkai Zasshi. 2018;122: $5-53$.

12. Inatani $M$, Higashide $\mathrm{T}$, Matsushita $\mathrm{K}$, et al. Intravitreal aflibercept in Japanese patients with neovascular glaucoma: the VEGA randomized clinical trial. Adv Ther. 2020. https://doi.org/10.1007/ s12325-020-01579-5.

13. Teich SA, Walsh JB. A grading system for iris neovascularization. Prognostic implications for treatment. Prognostic implications for treatment. Ophthalmology. 1981;88(11):1102-6. 